\title{
Lithium abundance dispersion in the Pleiades and M 34
}

\author{
P. Gondoin
}

\author{
European Space Agency, ESTEC - Postbus 299, 2200 AG Noordwijk, The Netherlands \\ e-mail: pgondoin@rssd.esa.int
}

Received 7 January 2014 / Accepted 5 March 2014

\begin{abstract}
Context. K-type stars of similar effective temperatures in clusters younger than about $250 \mathrm{Myr}$ are characterised by a wide dispersion in lithium abundance whose origin is not understood.

Aims. The present study addresses this question in light of rotation period measurements recently conducted in the Pleiades and M 34 open clusters.

Methods. The study investigates the dependence of lithium abundance with effective temperature distinguishing stars that belong to different rotation sequences in diagrams where measured rotation periods are plotted against $B-V$ colour indices.

Results. At any given effective temperature below $5500 \mathrm{~K}$, stars on the fast rotator sequence in the Pleiades and M 34 have significantly higher lithium abundances that stars with same masses and ages located on the slow rotator sequence.

Conclusions. This correlation suggests that shear-induced turbulence at the base of the convection zone accelerates the depletion of lithium in rapidly rotating late G- and K-type stars during a brief episode of strong rotational braking by stellar wind in their early evolution on the main sequence. The epoch at which this strong rotational braking occurs does not depend on stellar mass only but also on the rotation history of each individual star. I argue that the large dispersion in surface lithium abundance of $\mathrm{K}$ stars in the Pleiades and M 34 could be a consequence of this phenomenon.
\end{abstract}

Key words. open clusters and associations: general - stars: abundances - stars: rotation - stars: evolution - stars: solar-type stars: late-type

\section{Introduction}

The depletion of lithium and beryllium from the atmospheres of cool stars provides some information on the physical processes responsible for the redistribution of materials in their interiors. Predictions of the surface abundances of elements in stars are made by standard stellar models that include convection only as a mixing process. These models account for the decrease of lithium surface abundance with decreasing mass that have been observed in many open clusters including the Pleiades (e.g. Duncan \& Jones 1983) and the Hyades (e.g. Soderblom et al. 1995).

However, during the past two decades, observations of unevolved stars in open clusters and the field disagree with the predictions of the standard models (e.g. Randich 2006). In particular, K-type stars of similar effective temperatures in clusters younger than about $250 \mathrm{Myr}$ are characterised by a wide dispersion in $\mathrm{Li}$ abundance whose origin is not understood. As an example, the Pleiades show a large dispersion in surface Li abundance at a given colour for $T_{\text {eff }} \leq 5500 \mathrm{~K}$ (e.g., Duncan \& Jones 1983; Soderblom et al. 1993). On the contrary, G dwarfs in the Hyades cluster, much older than the Pleiades, exhibit little or no intrinsic dispersion in lithium abundance at a given mass. A similar behaviour is found in the Praesaepe star cluster (Soderblom et al. 1993), which has the same age and metallicity as the Hyades.

These observational results, not expected from standard models, suggest that Li depletion is not driven uniquely by convection, and that extra-mixing processes not included in classical theory are at work in stars during their early evolution. Tendencies for stars with high projected equatorial velocities to be lithium rich have been found in young clusters such as the Pleiades (Butler et al. 1987; Soderblom et al. 1993) and $\alpha$ Per (Balachandran et al. 1988) indicating that stellar rotation play an important role in these extra-mixing mechanisms (e.g. Chaboyer et al. 1995b; Montalban \& Schatzman 2000). Alternatively, King et al. (2010) suggested that the real abundance component to the Pleiades Li dispersion is the manifestation of relic differences in erstwhile pre-main-sequence Li burning caused by effects of surface activity on stellar structure.

Recently, photometric monitoring programmes have produced a large number of rotation period measurements in open clusters, including the Pleiades (Hartman et al. 2010) and M34 (Irwin et al. 2006; James et al. 2010; Meibom et al. 2011). These studies provide an opportunity to remove ambiguities associated with the use of projected equatorial velocity in the study of open clusters. These measurements have also shown (e.g., Barnes 2003; Meibom et al. 2009, 2011) that members of young stellar clusters tend to group on narrow sequences in diagrams where the measured rotation periods are plotted against masses or $B-V$ colour indices.

The present study revisits the connection between Li abundance and rotation in the Pleiades and M 34 open clusters in light of these new rotation rate measurements. The lithium and rotation datasets and the properties of the sample stars are reported in Sect. 2. Section 3 presents a classification of the sample stars into 

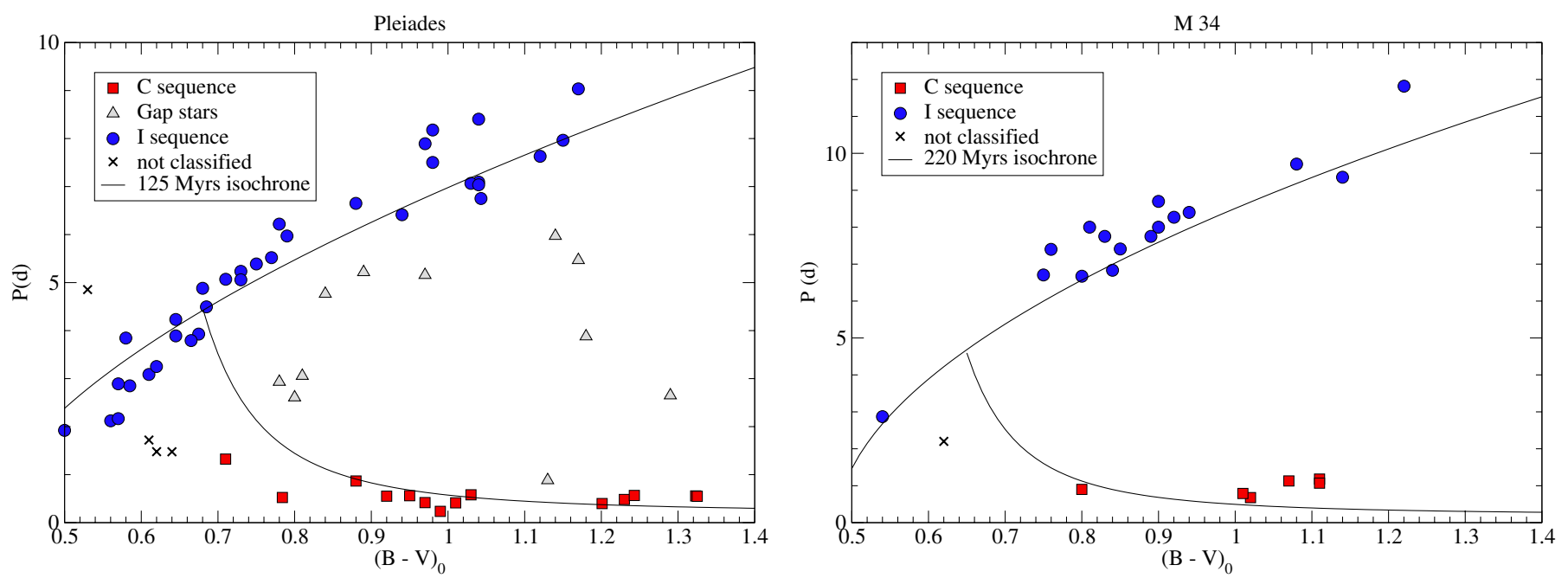

Fig. 1. Rotation periods of the Pleiades (left) and M34 (right) sample stars as a function of their $(B-V)_{0}$ colour indices compared with C and I isochrones.

rotation sequences and reports on their correlations with lithium abundances in the Pleiades and M 34 open clusters. The study results are discussed in Sect. 4.

\section{Compilation of the sample}

The stellar sample used in the present study consists of mainsequence stars with known lithium abundances and rotation periods that belong to the Pleiades and M34 open clusters.

Recently, Hartman et al. (2010) conducted an extensive photometric time-series survey of the Pleiades based on the membership list of Stauffer et al. (2007). Rotation periods were measured on 368 stars with $0.4 \leq M / M_{\odot} \leq 1.3$. I cross-matched these data with lithium abundance measurements in the Pleiades retrieved by King et al. (2000; see Table 1). These authors compile a list of 76 Pleiades members with known Li abundances from studies of Soderblom et al. (1993) and Jones et al. (1996) excluding binary systems and all stars with upper limits on the $\lambda 6707 \mathrm{Li}$ I line's equivalent width. Another star (HII 1653) classified as a spectroscopic binary by Hartman et al. (2010) was also excluded from the study. The colour indices of the remaining 64 sample stars are in the range $0.5<(B-V)_{0}<1.33$ which corresponds to late-F, $\mathrm{G}$, and early-K spectral types and to masses between 0.7 and $1.3 M_{\odot}$. All these stars have reached the main sequence since their Kelvin-Helmholtz contraction timescales is shorter than their age ( 100-150 Myr, Stauffer 2010).

Lithium abundances in M34 have been derived by Jones et al. (1997; see their Table 1) from a high resolution spectroscopy study of stars with high membership probabilities. I correlated these data with the list of 83 kinematic and photometric late-type M 34 cluster members with known rotation periods established by Meibom et al. (2011). Fourteen correlations were found. The lithium abundance measurements were also correlated with the results of a time series photometric survey of M 34 reported by Irwin et al. (2006). These authors measured the rotation periods of 105 cluster members selected from a $V$ vs. $V-I$ colour-magnitude diagram covering a range $14<V<24$. Twelve correlations were found of which 6 are new. Finally, the list of M 34 stars with known lithium abundance was correlated with the list of 55 solar-type stars in M 34, whose rotation periods were derived from differential photometry by James et al. (2010). Three new correlations were found. In total, 23 stellar members of the M34 open cluster have been found that have known rotational periods and $\mathrm{Li}$ abundances. Their colour indices in the range $0.55<(B-V)_{0}<1.23$ and age estimate between 177 Myr (Meynet et al. 1993) and 251 Myr (Ianna \& Schlemmer 1993) indicate that these stars have reached the main sequence.

\section{Analysis}

\subsection{Classification into rotation sequences}

Observations of main sequence stars with $\mathrm{G}$ and $\mathrm{K}$ spectral types in young open clusters $(\sim 100-200 \mathrm{Myr})$ show clear evidence for two distinct sequences of fast and slow rotators in period vs. mass or $(B-V)$ colour index diagrams (e.g. Hartman et al. 2010). These rotation sequences are called hereafter the (convective) $C$ sequence and the (interface) I sequence along the paradigm advanced by Barnes (2003).

Figure 1 (right) plots the rotational periods $P$ of the M34 sample stars as a function of their reddening corrected $(B-$ $V)_{0}$ indices. It also displays functional forms of the I and $\mathrm{C}$ sequences that were first introduced by Barnes (2003). For the I sequence, I used the form subsequently modified by Barnes (2007) in line with the gyrochronology analysis of M 34 performed by Meibom et al. (2011). The equations of these isochrones are as follows:

$$
\begin{aligned}
P_{\mathrm{C}} & =0.2 \exp \left(\left[(B-V)_{0}+0.1-t / 3000\right]^{3} \times t / 100\right) \\
P_{\mathrm{I}} & =\left[a \times\left((B-V)_{0}-b\right)^{c}\right] \times t^{n}
\end{aligned}
$$

where $a=0.730, b=0.481$, and $c=0.532$ are the coefficients determined from a least squares fit of $P_{\mathrm{I}}(t, B-V)$ to the M 34 I sequence using $n=0.52$ and $t=220$ Myr (Meibom et al. 2011). The value $n=0.52$ had been previously determined by demanding solar rotation at solar age using the I sequence from multiple young open clusters (Barnes 2007). From the data point positions relative to these curves, the M 34 sample stars were classified as lying on the $\mathrm{C}$ sequence, on the I sequence, or in the gap between these two sequences.

Hartman et al. (2010) compared the mass-period diagrams, which are equivalent to colour-period diagrams, of the Pleiades and four other open clusters with similar ages. They noted that the mass-period diagram of the Pleiades is remarkably similar to that seen by Meibom et al. (2009) for the M 35 open cluster 

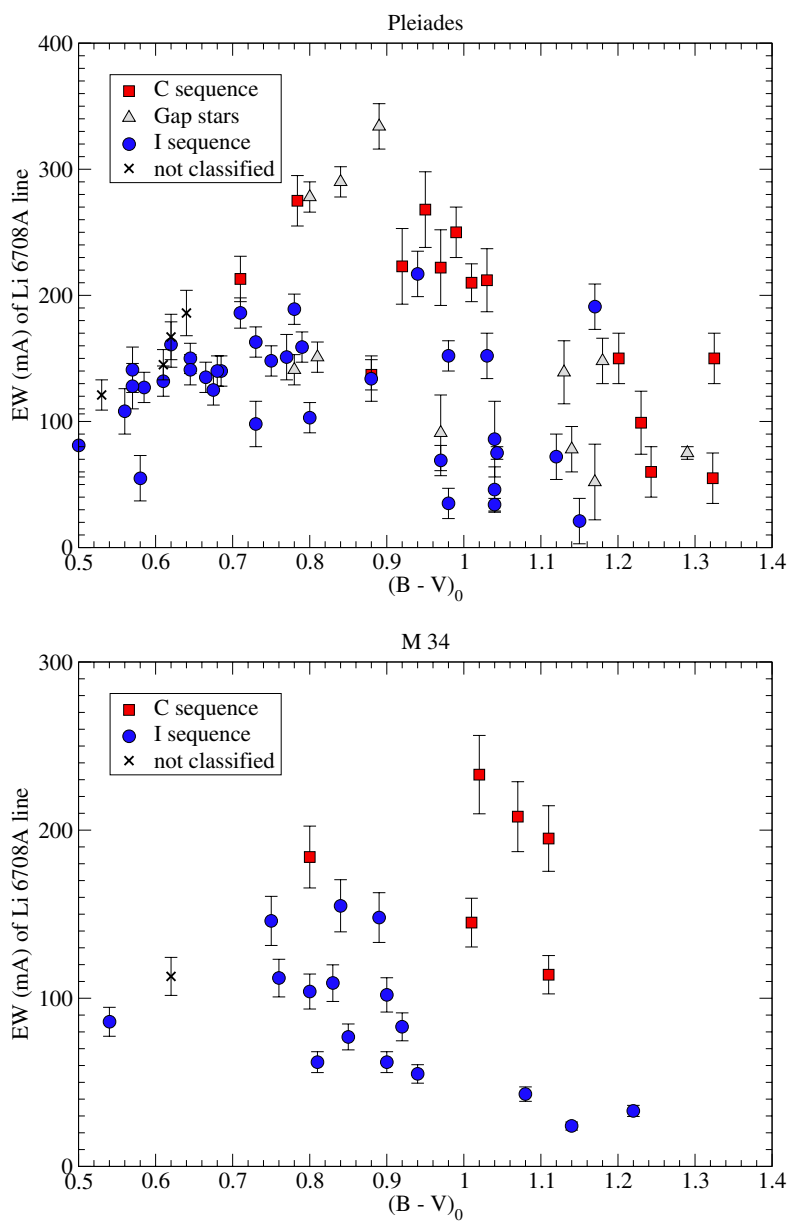

Fig. 2. Equivalent width of the Li $6708 \AA$ A line as a function of the $(B-$ $V)_{0}$ index corrected from reddening for the Pleiades (top) and M 34 (bottom) sample stars.

which gyro-age ranges from 134 Myr to 161 Myr. Figure 1 (left) shows the colour-period diagram of the Pleiades sample stars with known Li abundances. It also displays functional forms of the I and $\mathrm{C}$ rotation isochrones derived from the gyrochronology analysis of M 35 performed by Meibom et al. (2009). These forms are given by Eqs. (1) and (2) with $a=0.77, b=0.40$, $c=0.60$, and $n=0.52$. I classified the Pleiades sample stars as members of the $\mathrm{C}$ sequence, of the I sequence or of the gap based on their proximity to the $125 \mathrm{Myr} \mathrm{C}$ and I isochrones.

Four stars in the Pleiades and one star in M 34 with $(B-V)_{0}<$ 0.7 could not be classified and are marked by crosses in Fig. 1 . These blue and bright objects are most likely close binaries in which the active component is locked into high rotation by synchronisation with the orbital period due to tidal effects.

\subsection{Lithium abundance and rotation}

The Li I $\lambda 6707.8 \AA$ equivalent widths of the Pleiades and M34 sample stars are plotted vs. their reddening corrected $(B-V)_{0}$ indices in the upper and lower diagrams of Fig. 2.

The Li spectral feature encompasses a blending complex that lies some $0.4 \AA$ blueward of the $\mathrm{Li}$ doublet. Its contribution of about $10 \mathrm{~m} \AA$ is significantly smaller than the typical $\mathrm{Li}$ line strength that ranges between 50 to $300 \mathrm{~m} \AA$. It was subtracted in the Pleiades sample stars by King et al. (2000) following the approach of Soderblom et al. (1993), who parameterised the contaminating line strength as a function of the $B-V$ colour index. King et al. (2000) estimated that the uncertainties on the Li I $\lambda 6707.8 \AA$ line equivalent width measurements are in the range 5 to $30 \mathrm{~m} \AA$ based on measurement errors of Soderblom et al. (1993) and Jones et al. (1996) that are dependent on projected rotational velocities. Jones et al. (1997) found a comparable 10\% uncertainty in M 34 from several independent measurements of the Li equivalent width in each star.

Figure 2 shows that, for $(B-V)_{0}>0.7$, the dispersion in equivalent width of the $\mathrm{Li}$ I $\lambda 6707.8 \AA$ line is significantly higher than the measurement errors. It also reveals that, in this colour index range, the $\mathrm{Li}$ line equivalent widths of $\mathrm{C}$ sequence and gap stars are systematically larger than those of I sequence stars.

Soderblom et al. (1993) calculated lithium abundances in the Pleiades from the Li I $\lambda 6707.8 \AA$ line equivalent widths values using conventional LTE curves of growth. The derived abundances were tabulated as a function of the line equivalent widths and the star effective temperature. King et al. (2000) updated previous Li abundances derivation by applying this tabulation to corrected Li line strengths and effective temperatures values derived from $B-V$ and $V-I$ colour indices. They derived the uncertainties on the final abundances by summing in quadrature uncertainties in the Li line measurements and on the effective temperatures. These errors on the Li abundance of the Pleiades sample stars typically range from 0.1 to 0.26 dex with the exception of the HII 1110 and HII 3019 stars which have very low Li abundances. Jones et al. (1997) estimated that a $10 \%$ error in equivalent width lead to an abundance error of about 0.05 to 0.15 dex. Further errors in temperatures due to errors in the colours give errors in abundances of 0.1 to 0.2 dex.

The lithium abundances of the Pleiades and M34 sample stars are plotted as a function of their effective temperatures in the upper and lower diagrams of Fig. 3. The lithium abundance decreases with effective temperature by nearly three orders of magnitude between $6000 \mathrm{~K}$ and $4000 \mathrm{~K}$.

This well-known decay is expected from standard models of stellar evolution that predict the attrition of $\mathrm{Li}$ as a function of stellar mass, and age. According to these models, pre-main sequence stars with low masses evolving down the Hayashi track develop high enough temperatures at the base of their convection zone for energetic protons to destroy Li there, thereby depleting their surface Li abundance. Reversely, little Li depletion is expected for stars reaching the zero-age main sequence (ZAMS) as A, F, or G dwarfs (e.g., Pinsonneault 1997). Since Li depletion ceases as the surface convection zone recedes towards the surface and its base cools, standard models of stellar evolution also predicts that no further $\mathrm{Li}$ depletion occurs on the mainsequence for A, F, and G dwarfs (e.g., Proffitt \& Michaud 1989; Deliyannis et al. 1990; Swenson et al. 1994), whereas K and $\mathrm{M}$ dwarfs should continue to deplete Li as they evolve beyond the ZAMS. This prediction is consistent with Fig. 3. Indeed, G-type stars have similar Li abundance in the Pleiades and M 34 while K-type stars have in average lower $\mathrm{Li}$ abundance in the older M34 cluster. This comparison is justified since the two clusters have similar metallicities (Schuler et al. 2003; Taylor 2008).

However, standard models also predict that ${ }^{7} \mathrm{Li}$ depletion in a star is a unique function of age, mass, and metallicity. Figure 3, in contrast, shows that stars with the same $T_{\text {eff }}$ in the Pleiades or in M 34 have significantly different Li abundances. The scatter in the star-to-star $\mathrm{Li}$ abundance is particularly large in the 5500-4000 K temperature range as noted by Jones et al. (1997) in M 34 and by Soderblom et al. (1993), Jones et al. (1996), 

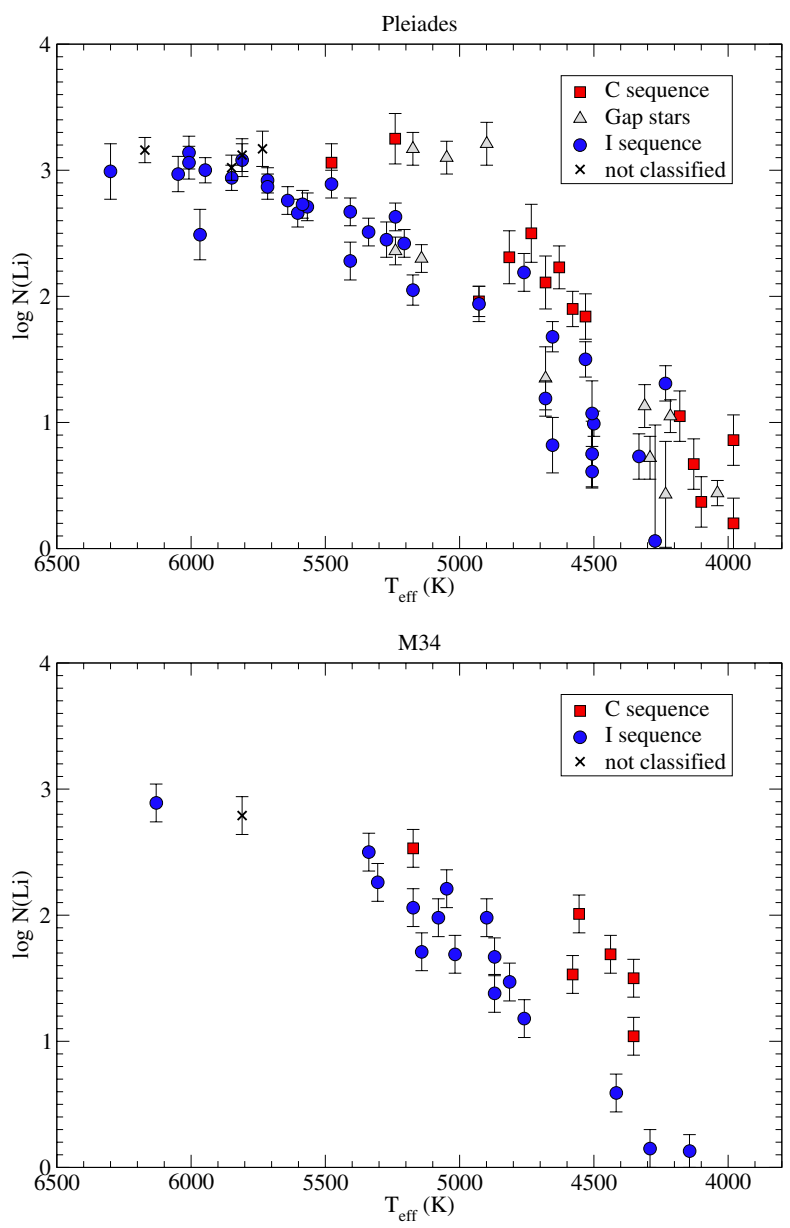

Fig. 3. Lithium abundance vs. effective temperature for the Pleiades (top) and M 34 (bottom) sample stars.

and King et al. (2000) in the Pleiades. A comparison of the observed scatter in Li abundances with the estimated uncertainties indicates that this spread is statistically significant. Furthermore, Fig. 3 shows that, at any given temperature below $5500 \mathrm{~K}, \mathrm{C}$ sequence and gap stars in the Pleiades have significantly higher lithium abundances than I sequence stars with the same effective temperature. This confirms the correlation between rotation sequences and Li abundance suggested in Fig. 2.

\section{Discussion}

Independently from any specific model of stellar rotation evolution, the clustering of young main-sequence stars into fast and slow rotation sequences in period vs. colour index diagrams, their later evolution through the gap towards a single sequence, and the low density of stars in the gap indicate that stars spend less time in this part of the diagram than on the $\mathrm{C}$ and $\mathrm{I}$ sequences. The narrow $\mathrm{C}$ sequence in the Pleiades and M 34 also suggests that the rapidly rotating $\mathrm{C}$ stars loose little angular momentum until they quickly evolve through the gap onto the I sequence. The transition from the $\mathrm{C}$ to the I sequence thus constitutes an evidence for a brief phase of strong surface rotation deceleration among rapidly rotating late- $\mathrm{G}$ and $\mathrm{K}$ type stars in their early phase of evolution on the main sequence.

This interpretation is supported by models of stellar rotation evolution that predict a large deceleration of rotation among rapidly rotating solar-type stars during their first few $100 \mathrm{Myr}$ on the main sequence (Gallet \& Bouvier 2013). The epoch at which this strong rotational braking occurs does not depend on mass only, since main sequence stars in young open cluster with a same mass and age can be either on the $\mathrm{C}$ sequence, on the I sequence or in the gap. This epoch also depends on other stellar parameters such as the initial rotation period on the ZAMS (Barnes 2010) which itself depends on the rotation history of each star during its pre-main sequence contraction (see Bouvier et al. 2013, and reference therein).

While the empirical model of Barnes (2010) explains the positions and shapes of the rotation sequences across the observed colour-period diagrams, simplified models of angular momentum redistribution based on a double-zone model appear to grasp the main trends of the rotation evolution of solar-type stars (Gallet \& Bouvier 2013). This includes the spin-up during pre-main sequence contraction, the rapid spin-down near the ZAMS, and the convergence of surface rotation rates during further evolution on the main sequence. However, these models do not describe the differential rotation in stellar interiors. The double-zone model originally proposed by MacGregor (1991) is a crude approximation of actual internal rotational profiles. The evolution of internal rotation as the star ages is also sensitive to the assumed braking law. Quantitative inferences on the effect of a rapid rotation deceleration on lithium depletion require a physical modelling of the angular momentum loss due to stellar winds and of the redistribution of angular momentum in stellar interiors.

The fact that the transition from the $\mathrm{C}$ to the I rotation sequence in the Pleiades and M 34 is correlated with a drop in $\mathrm{Li}$ abundance suggests that the associated decay of the rotation rate is accelerating the depletion of lithium in young late- $\mathrm{G}$ and K-type stars. Rotational braking by stellar winds is the commonly accepted explanation for the decay of stellar rotation on the main sequence. Since the magnetic field lines that sling charged particles from the wind into space are rooted in the photosphere, the wind torque is expected to decelerate the envelope rotation while the conservation of angular momentum should keep the radiative core in rapid rotation. Gap stars in young open clusters should thus develop a large shear at the base of their convection zone that triggers various instabilities. Studies indicate that these instabilities drive mass motions or gravity waves that redistribute angular momentum and mix the stellar material enhancing light-element depletion (e.g. Chaboyer et al. 1995b; Charbonnel \& Talon 2005; Talon 2008).

The dispersion in surface $\mathrm{Li}$ abundance observed among $\mathrm{K}$ stars in young open clusters could thus result from an acceleration of the Li depletion in rapidly rotating $\mathrm{K}$ stars due to shear instabilities at the bottom of the convection zone during a brief episode of strong rotational braking by stellar wind in their early evolution on the main sequence. This scenario explains that no lithium dispersion is observed in the Hyades and Praesaepe star clusters (Soderblom et al. 1993) since, by the age of these clusters $(\sim 700 \mathrm{Myr})$, observations show a clear convergence in the angular momentum evolution of all F, G, and $\mathrm{K}$ dwarfs towards a single sequence of slow rotators. All the stars have by then experienced a strong rotational braking that accelerated Li depletion and the coolest stars $\left(T_{\text {eff }}<5000 \mathrm{~K}\right)$ have fully depleted their lithium.

Acknowledgements. I am grateful to the anonymous referee for the helpful comments that allowed me to improve the paper.

\section{References}

Balachandran, S., Lambert, D. L., \& Stauffer, J. R. 1988, ApJ, 133, 267 Barnes, S. A. 2003, ApJ, 586, 464 
Barnes, S. A. 2007, ApJ, 669, 1167

Barnes, S. A. 2010, ApJ, 722, 222

Bouvier, J., Matt, S. P., Mohanty, S., et al. 2013, in Protostars \& Planets VI, eds. H. Beuther, R. Klessen, K. Dullemond, \& Th. Henning (University of Arizona Press), accepted [arXiv: 1309.7851]

Butler, R. P., Marcy, G. W., Cohen, R. D., \& Duncan, D. K. 1987, ApJ, 319, L19

Chaboyer, B., Demarque, P., \& Pinsonneault, M. H. 1995, ApJ, 441, 876

Charbonnel, C. \& Talon, S. 2005, Science, 309, 2189

Deliyannis, C. P., Demarque, P., \& Kawaler, S. D. 1990, ApJS, 73, 21

Duncan, D. K., \& Jones, B. F. 1983, ApJ, 271, 663

Gallet, F., \& Bouvier, J. 2013, A\&A, 556, A36

Hartman, J. D., Bakos, G. A., Kovács, G., \& Noyes, R. W. 2010, MNRAS, 408, 475

Ianna, P. A., \& Schlemmer, D. M. 1993, AJ, 105, 209

Irwin, J., Aigrain, S., Hodgkin, S., et al. 2006, MNRAS, 370, 954

James, D. J., Barnes, S. A., Meibom, S., et al. 2010, A\&A, 515, A100

Jones, B. F., Shetrone, M., Fischer, D., \& Soderblom, D. R. 1996, AJ, 112, 186

Jones, B. F., Fischer, D., Shetrone, M., \& Soderblom, D. R. 1997, AJ, 114, 352

King, J. R., Krishnamurthi, A., \& Pinsonneault, M. H. 2000, AJ, 119, 859

King, J. R., Schuler, S. C., Hobbs, L. M., \& Pinsonneault, M. H. 2010, ApJ, 710, 1610
MacGregor, K. B. 1991, in Angular Momentum Evolution of Young Stars, eds. S. Catalano, \& J. R. Stauffer (Dordrecht: Kluwer), 315

Meibom, S., Mathieu, R. D., \& Stassun, K. G. 2009, ApJ, 695, 679

Meibom, S., Matthieu, R. D., Stassun, K. G., et al. 2011, ApJ, 733, 115

Meynet, G., Mermilliod, J., \& Maeder, A. 1993, A\&AS, 98, 477

Montalban, J., \& Schatzman, E. 2000, A\&A, 354, 943

Pinsonneault, M. 1997, ARA\&A, 35, 557

Proffitt, C. R., \& Michaud, G. 1989, ApJ, 346, 976

Randich, S. 2006, in Chemical Abundances and Mixing in Stars in the Milky Way and its Satellites, ESO Astrophysics Symposia (Springer-Verlag), 173 Schuler, S. C., King, J. R., Fischer, D. A., et al. 2003, AJ, 125, 2085

Soderblom, D. R., Jones, B. F., Balachandran, S., et al. 1993a, AJ, 106, 1059 Soderblom, D. R., Fedele, S. B., Jones, B. F., et al. 1993b AJ, 106, 1080 Soderblom, D. R., Jones, B. F., Stauffer, J. R., \& Chaboyer, B. 1995, AJ, 110, 729

Stauffer, J. R. 2010, in Star Clusters in the Era of Large Survey, eds. A. Moitinho, \& J. A. Alves, Proc. Symp. 5 of JENAM, 155

Stauffer, J. R., Hartmann, L. W., Fazio, G. G., et al. 2007, ApJS, 172, 663

Swenson, F. J., Faulkner, J., Rogers, F. J., \& Iglesias, C. A. 1994, ApJ, 425, 286

Talon, S. 2008, Mem. Soc. Astron. It., 79, 569

Taylor, B. J. 2008, AJ, 136, 1388 